\title{
Utility of cine MRI in evaluation of cardiovascular invasion by mediastinal
} masses

\author{
Sourav Panda, Aparna Irodi, Riya Daniel, Binita R Chacko, Leena R Vimala, \\ Birla R Gnanamuthu \\ Departments of Radiology and ${ }^{1}$ Cardiothoracic Surgery, Christian Medical College, Vellore, Tamil Nadu, India \\ Correspondence: Prof. Aparna Irodi, Department of Radiology, Christian Medical College, Vellore - 632004, Tamil Nadu, India. \\ E-mail: aparnashyam@gmail.com
}

\section{Abstract}

Background: Accurate imaging assessment of cardiovascular invasion by mediastinal masses is essential for determining surgical feasibility. This can sometimes be difficult on CT owing to limited space available in the mediastinum, resulting in mediastinal masses abutting and indenting adjacent cardiovascular structures. Cine MRI may aid in such situations by demonstrating differential mobility. Aims and Objectives: To evaluate the role of cine MRI in assessing cardiovascular invasion by mediastinal masses, by evaluating sliding motion and the presence of chemical shift artifact between the mediastinal mass and apposing structures. Material and Methods: Retrospective study of 44 patients with mediastinal masses, with equivocal involvement of 162 cardiovascular structures on CT scan, in whom cine MRI was done. Involvement on CT was considered equivocal when there was a loss of intervening fat plane and broad surface $(>3 \mathrm{~cm})$ or angle $\left(>90^{\circ}\right)$ of contact between the mediastinal mass and cardiovascular structure. The presence of either sliding movement or type 2 chemical shift artifact or both between mass and the cardiovascular structure was considered as no adherence or invasion. The absence of both the parameters was considered as the presence of invasion or adhesion. Imaging findings were correlated with intraoperative findings. Results: After excluding 25 cardiovascular structures in 7 patients, 137 cardiovascular structures whose involvement was suspected on CT were evaluated in 37 patients with mediastinal masses. In all, 31 cardiovascular structures showed invasion on MRI out of which 28 structures were invaded or adhered intraoperatively and 106 cardiovascular structures showed no invasion on MRI out of which 97 structures were intraoperatively not invaded/adhered. The sensitivity, specificity and accuracy of our study are $75.7 \%, 97 \%$ and $91.2 \%$, respectively. Conclusion: Cine MRI can be used as an effective tool in patients with equivocal cardiovascular invasion by mediastinal masses on CT scans.

Key words: Cardiovascular invasion; cine MRI; mediastinal masses

\section{Introduction}

Accurate assessment of the local extent of mediastinal masses is essential to determine surgical feasibility and

\begin{tabular}{|l|l|}
\hline \multicolumn{2}{|c|}{ Videos Available on: www.ijri.org } \\
\hline \multicolumn{2}{|c|}{ Access this article online } \\
\hline Quick Response Code: & Website: \\
\hline www.ijri.org
\end{tabular}

decide upon the future course of management. Owing to limited space available in the mediastinum, masses

This is an open access journal, and articles are distributed under the terms of the Creative Commons Attribution-NonCommercial-ShareAlike 4.0 License, which allows others to remix, tweak, and build upon the work non-commercially, as long as appropriate credit is given and the new creations are licensed under the identical terms.

For reprints contact: WKHLRPMedknow_reprints@wolterskluwer.com

Cite this article as: Panda S, Irodi A, Daniel R, Chacko BR, Vimala LR, Gnanamuthu BR. Utility of cine MRI in evaluation of cardiovascular invasion by mediastinal masses. Indian J Radiol Imaging 2020;30:280-5.

Received: 02-Apr-2020 Accepted: $21-J u n-2020$

Revised: 10-Jun-2020 Published: $15-$ Oct- 2020 
appear to abut the adjacent cardiovascular structures and sometimes it becomes difficult on contrast-enhanced CT scans to confirm or rule out cardiovascular invasion by mediastinal masses, often over-diagnosing possible infiltration of cardiovascular structures, thus denying patients of potentially curative surgery.

Currently, contrast-enhanced CT scans are most commonly used for preoperative assessment of mediastinal masses. Some criteriathat have been studied for diagnosing local mediastinal invasion on CT include loss of intervening fat planes, broad surface of contact, and a large angle of contact $\left(90^{\circ}\right.$ or $\left.180^{\circ}\right)$ between the mass and adjacent cardiovascular structures, indentation or distortion of vessel contour and intraluminal extension. ${ }^{[1-3]}$ However, the sensitivity, specificity and accuracy of CT scan for cardiovascular invasion vary in literature and largely depend upon the study design and patient population. Sensitivity and specificity ranges are $40 \%-77.8 \%$ and $67 \%-99 \%$, respectively. ${ }^{[4,5]}$

We aimed to evaluate the additional role of steady-state free precession (SSFP) cine MRI to visualize the differential sliding motion and presence or absence of type 2 chemical shift artefact (black boundary artefact) between the mediastinal mass and adjacent cardiovascular structures in patients with equivocal findings of invasion on CT scan.

\section{Materials and Methods}

A retrospective study of 44 patients was performed between 2010 and 2018 for who had undergone cine SSFP MRI to evaluate equivocal findings of cardiovascular invasion by mediastinal masses on CT scan. The imaging findings were correlated with intraoperative findings. Seven patients were excluded from the study as they were deemed inoperable, in view of extensive infiltration on imaging or detection of metastases.

\section{CT Technique and interpretation}

Contrast-enhanced CT scans of the thorax in axial and coronal planes with slice thickness $2.5-5 \mathrm{~mm}$ using $1 \mathrm{ml} / \mathrm{kg}$ of non-ionic iodinated contrast media were obtained using 16 slice Siemens Emotion or 64 slice GE Discovery CT750 HD CT scanners.

CT findings were considered equivocal for cardiovascular invasion when there was a loss of intervening fat planes, broad surface of contact $(>3 \mathrm{~cm})$ or $>90^{\circ}$ angle of contact between the mass and adjacent cardiovascular structures [Figure 1A and B] or in a multidisciplinary team meeting if the reporting radiologist and thoracic surgeon had any suspicion of infiltration.

\section{MRI Technique and interpretation}

The MRI scans were performed on a 1.5-Tesla Siemens Avanto scanner. Bright blood SSFP non-cine images were
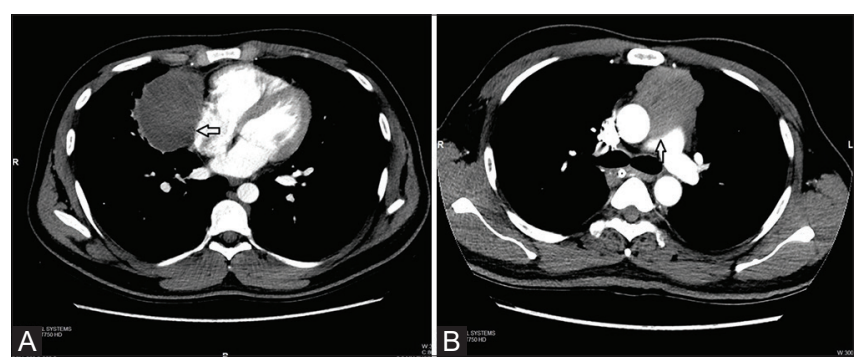

Figure 1 (A and $B$ ): (A) Axial CECT images illustrate the criteria used to define equivocal cardiovascular invasion on CT. (A) shows a broad surface of contact $(>3 \mathrm{~cm})$ and obliteration of intervening fat plane between mass and pericardium (arrow). (B) shows $>90^{\circ}$ angle of contact and loss of intervening fat plane between mass and ascending aorta (arrow)

obtained at repetition time/echo time (TR/TE) of 290-360 $\mathrm{ms} / 1.1-1.4 \mathrm{~ms}$, with a slice thickness of $6-8 \mathrm{~mm}$, slice spacing of 6.3-6.5 mm, field of view (FOV) ranging from 340 to $350 \mathrm{~mm}$, matrix ranging from $240-300 \times 208-256$, receiver bandwidth of $866 \mathrm{~Hz} / \mathrm{Px}$, the phase-encoding direction was anteroposterior and frequency-encoding direction was right to left. Cardiac-gated cine SSFP images were obtained at TR/TE of 29-41 ms/1.1-1.3 ms with a slice thickness of 6-8 mm, FOV ranging from 340 to $350 \mathrm{~mm}$, matrix of 192-256 × 208-256, receiver bandwidth of $930 \mathrm{~Hz} /$ Px, phase-encoding direction was antero-posterior and frequency-encoding direction was right to left. Cine and non-cine images were taken in axial, coronal and sagittal planes. In most patients, oblique coronal and oblique sagittal cine images were also acquired, with the plane of imaging perpendicular to the interface between mass and cardiovascular structure being studied.

The presence or absence of type 2 chemical shift artifact was evaluated on both cine and non-cine images [Figure 2A and B] and differential sliding motion between the mass and adjacent cardiovascular structure was assessed on cine images [Figure 3A, B and Video 1]. These two parameters were used on MRI to assess invasion or adhesion of mediastinal masses to the cardiovascular structures.

The presence of either sliding motion or chemical shift artifact or the presence of both was considered as indicating no invasion or adhesion. The absence of both differential motion and chemical shift artifact was interpreted as invasion or adhesion.

\section{Results}

Demographics

Forty-four patients with mediastinal masses who had undergone additional MRI to evaluate equivocal findings on CT were included. There were 31 males and 13 females; mean age 36.5 years; range 11-65 years. A total of 162 cardiovascular structures showed doubtful findings on CT. 
Imaging findings were correlated with surgical findings in 137 cardiovascular structures in 37 patients. In all, 25 cardiovascular structures in 7 patients were excluded as they did not undergo surgery owing to extensive infiltration or presence of metastases on imaging.

\section{Diagnoses}

A wide variety of histopathological diagnoses was encountered. Thymic lesions were the commonest including thymoma $(n=10)$, thymic carcinoma $(n=3)$ and thymic carcinoid $(n=3)$, others included malignant germ cell tumors $(n=5)$, mature teratoma $(n=3)$, sarcoma $(n=2)$, benign cysts $(n=4)$, neurogenic tumors $(n=5)$, inflammatory myofibroblastic tumor $(n=1)$ and lymphoma $(n=1)$.

\section{MRI findings and correlation with intraoperative findings}

A total of 137 cardiovascular structures with equivocal invasion on CT scan in the 37 patients were evaluated on MRI for presence of sliding motion and chemical shift artifact. Pericardium $(n=31)$ was the most common structure evaluated followed by aorta $(n=19)$. Other structures included main pulmonary artery $(n=16)$, right/left pulmonary arteries $(n=11)$, superior vena cava $(n=14)$, atria $(n=18)$, brachiocephalic vein $(n=11)$, pulmonary veins $(n=8)$,

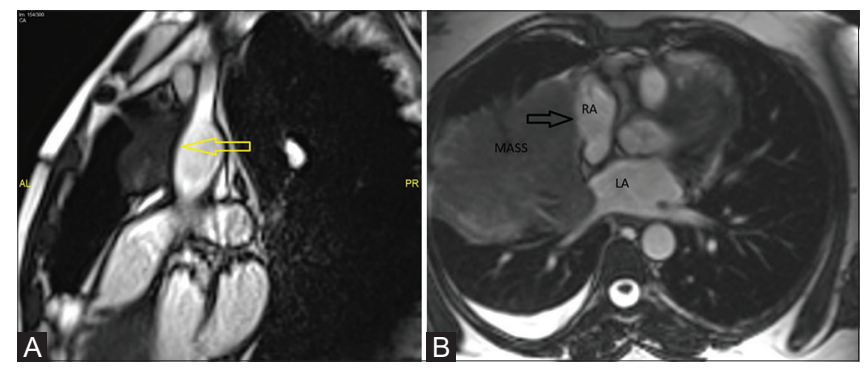

Figure 2 (A and B): (A) Non-cine sagittal image show the presence (A) of chemical shift artifact between the mediastinal mass and ascending aorta (arrow). (B) Non-cine axial SSFP images show the absence (B) of chemical shift artifact between mediastinal mass and right atrium (arrow) ventricles $(n=4)$, inferior vena cava $(n=3)$, left subclavian artery $(n=1)$ and left common carotid artery $(n=1)$.

There was no invasion or adhesion in 106 cardiovascular structures on MRI while 31 structures showed invasion or adhesion on imaging. Intraoperatively, 38 cardiovascular structures were involved and 99 structures were not invaded or adhered to.

Out of 31 structures that showed features of invasion or adhesion on MRI, 28 structures were intraoperatively invaded (true positive for 'invasion') [Figures 4, 5 and Videos 2, 3] and three structures were free (false positive for 'invasion'). Out of 106 structures showing no invasion on MRI, 97 were intraoperatively not invaded or adhered (true negative) [Figure 6 and Video 4] and 9 structures were involved (false negative). Details of the status of involvement of individual cardiovascular structures on imaging and surgery is illustrated in Table 1.

Using our imaging criteria for invasion, cine MRI was able to exclude or confirm invasion of cardiovascular structures by mediastinal masses with sensitivity, specificity and
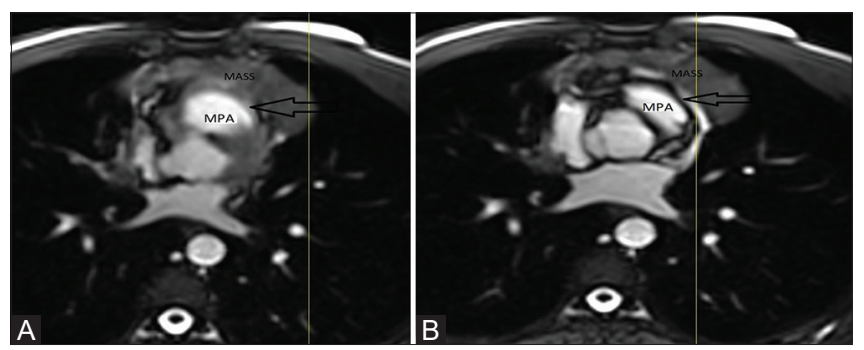

Figure 3 ( $A$ and $B$ ): (A) Limited images from cine axial SSFP images in end-systolic (A) shows differential sliding motion between the main pulmonary artery (MPA) and anterior mediastinal mass (arrow). (B) shows differential sliding motion between MPA and anterior mediastinal mass (arrow). A sliver of pericardial fluid seen between MPA and the mass in (B)

Table 1: Illustrates the status of infiltration of individual cardiovascular structures on imaging and surgery

\begin{tabular}{|c|c|c|c|c|c|c|c|c|}
\hline Cardiovascular Structure & $\begin{array}{c}\text { No Invasion/ } \\
\text { Adhesion on MRI }\end{array}$ & $\begin{array}{c}\text { Invaded/Adhered } \\
\text { on MRI }\end{array}$ & $\begin{array}{l}\text { Intraoperatively } \\
\text { Uninvolved }\end{array}$ & $\begin{array}{l}\text { Intraoperatively } \\
\text { Involved }\end{array}$ & $\begin{array}{c}\text { True } \\
\text { Negative }\end{array}$ & $\begin{array}{c}\text { False } \\
\text { Negative }\end{array}$ & $\begin{array}{c}\text { True } \\
\text { Positive }\end{array}$ & $\begin{array}{c}\text { False } \\
\text { Positive }\end{array}$ \\
\hline Aorta & 19 & 0 & 17 & 2 & 17 & 2 & 0 & 0 \\
\hline MPA & 15 & 1 & 15 & 1 & 14 & 1 & 0 & 1 \\
\hline RPA/LPA & 11 & 0 & 9 & 2 & 9 & 2 & 0 & 0 \\
\hline SVC & 7 & 7 & 7 & 7 & 7 & 0 & 7 & 0 \\
\hline Pericardium & 19 & 12 & 17 & 14 & 18 & 1 & 12 & 0 \\
\hline Atria & 18 & 0 & 16 & 2 & 16 & 2 & 0 & 0 \\
\hline Ventricles & 4 & 0 & 4 & 0 & 4 & 0 & 0 & 0 \\
\hline Pulmonary Veins & 8 & 0 & 7 & 1 & 7 & 1 & 0 & 0 \\
\hline Inferior venacava (IVC) & 2 & 1 & 2 & 1 & 2 & 0 & 1 & 0 \\
\hline Brachiocephalic vein (BCV) & 3 & 8 & 5 & 6 & 3 & 0 & 6 & 2 \\
\hline Aortic arch branches (left CCA and left SCA) & 0 & 2 & 0 & 2 & 0 & 0 & 2 & 0 \\
\hline Total & 106 & 31 & 99 & 38 & 97 & 9 & 28 & 3 \\
\hline
\end{tabular}




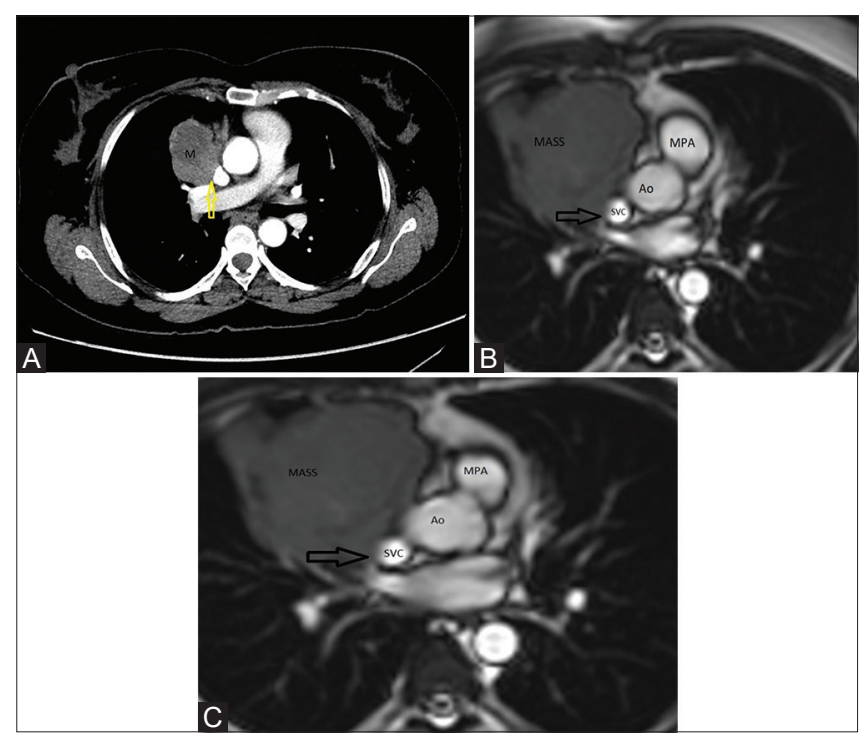

Figure 4 (A-C): (A) Axial CECT image (A) of a 39-year-old man with biopsy-proven thymic carcinoid showing obliteration of fat plane and $>90^{\circ}$ angle of contact between anterior mediastinal mass $(\mathrm{M})$ and superior vena cava (arrow). (B) End-systolic (B) and end-diastolic (C) limited cine axial SSFP images show the absence of chemical shift artifact and sliding motion between the mass and SVC, indicating invasion or adherence

accuracy of $75.7 \%, 97 \%$ and $91.2 \%$, respectively, in cases with equivocal invasion on the initial CECT scan.

All the cardiovascular structures with the presence of chemical shift artifact showed differential sliding motion except in one case involving pericardium, where sliding motion was absent due to the large pericardial effusion rather than actual invasion [Figure 7A, B and Video 5].

The sensitivity, specificity, and accuracy of sliding motion and chemical shift artifact as independent parameters to exclude or confirm cardiovascular invasion is shown in Table 2.

\section{Discussion}

Accurate assessment of cardiovascular invasion by mediastinal masses is essential to determine surgical feasibility and in planning the surgery. Many centers consider the invasion of the myocardium and great vessels as contraindications for surgical resection. ${ }^{[6]}$ Computed tomography has long been the modality of choice for pre-operative evaluation of mediastinal masses. However in some cases, the accurate assessment of cardiovascular invasion by intrathoracic masses becomes difficult on contrast-enhanced CT, due to the limited space in the mediastinum resulting in the mass indenting on adjacent structures even if it does not infiltrate.

MRI has found limited use in the evaluation of thoracic pathology as it is expensive, more time consuming than CT

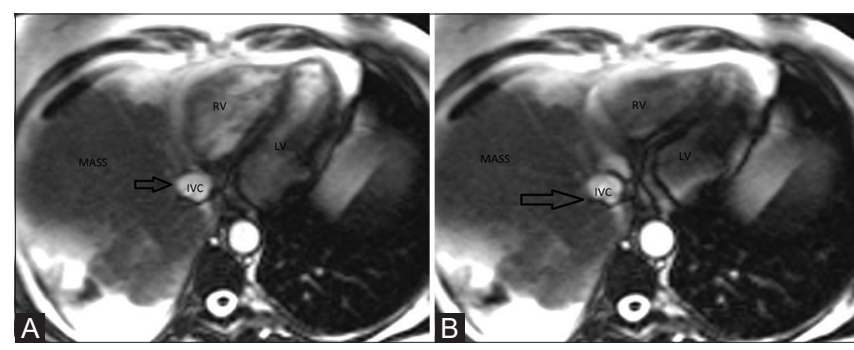

Figure $\mathbf{5}$ (A and $B$ ): (A) Limited cine axial MRI images of a 45-year-old lady with biopsy-proven invasive thymoma in end-diastolic (A) illustrating the loss of chemical shift artifact and absence of sliding motion between inferior vena cava (IVC) and the mediastinal mass, suggesting invasion/adhesion on MRI. Intraoperatively, mass was adherent to IVC. (B) Cine axial images of a 45-year-old lady with biopsy-proven invasive thymoma in end-systolic phases (B) illustrating the loss of chemical shift artifact and absence of sliding motion between inferior vena cava (IVC) and the mediastinal mass, suggesting invasion/ adhesion on MRI. Intraoperatively, the mass was adherent to IVC

Table 2: The sensitivity, specificity and accuracy of individual imaging criteria that has been used to confirm or exclude cardiovascular invasion

\begin{tabular}{lccc}
\hline Parameter & Sensitivity & Specificity & Accuracy \\
\hline Sliding motion & $75.6 \%$ & $96 \%$ & $90.5 \%$ \\
Chemical shift artifact & $75.7 \%$ & $97 \%$ & $91.2 \%$ \\
\hline
\end{tabular}

and lack of protons in lung tissue resulting in poor signal return. ${ }^{[7]}$ However, owing to higher soft-tissue resolution and the advent of improved and faster imaging techniques, including the ECG-gated fast GRE cine sequences, MRI can be used as an alternative or complimentary modality.$^{[8-10]}$ Cine images can be acquired using spoiled or incoherent GRE sequences (where the residual transverse magnetization is spoiled) and balanced steady state or coherent GRE sequences (where the transverse magnetization is refocused and added to the longitudinal magnetization to create a steady state). ${ }^{[11]}$ Balanced SSFP (bSSFP) sequences have a higher blood-tissue contrast but are more sensitive to the field inhomogeneties, which tend to increase with increasing magnetic strength. Hence, bSSFP sequences have become the method of choice to acquire cine images at low magnetic field strength $(1.5 \mathrm{~T})$ while spoiled GRE sequences, which are less sensitive to local field variations, are used at higher magnetic fields (3T or higher). ${ }^{[12]}$ In this study, we used bSSFP sequences to acquire the cine and non-cine images to evaluate the presence of chemical shift artifact and differential motion between mediastinal masses and adjacent cardiovascular structures.

Black line/black boundary artifact also called the type 2 chemical shift artifact is seen as a black line between the apposing structures in out of phase imaging like SSFP sequences and suggests an intact fat-water interface. ${ }^{[13-15]}$ Type 2 chemical shift artifact is already being used for delineating borders of organs surrounded by fat in other 


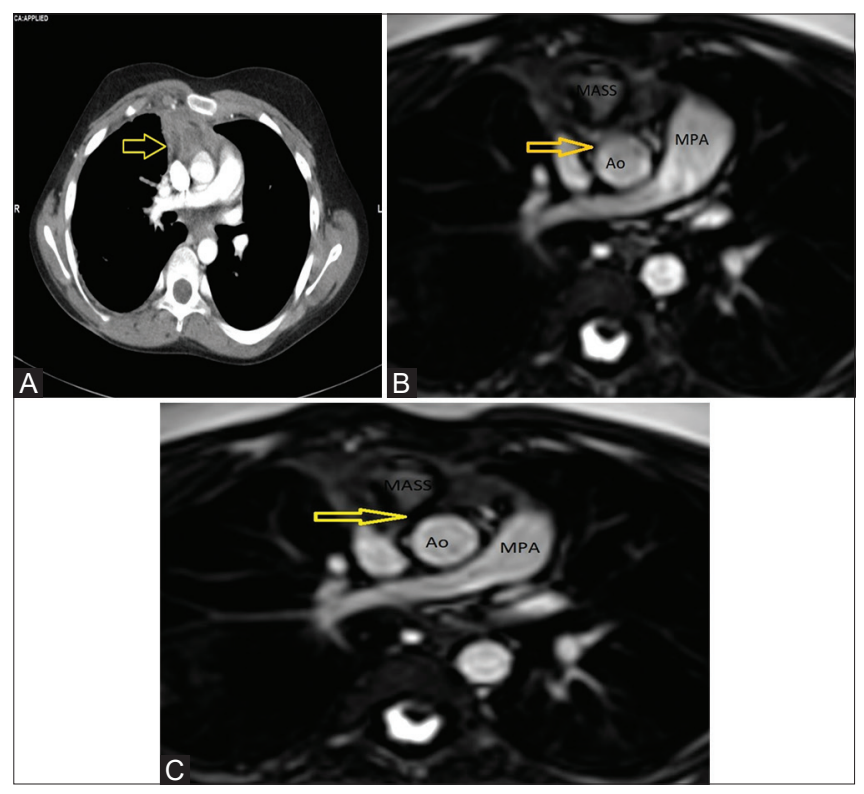

Figure 6 (A-C): (A) Axial CECT image of an 11-year-old boy with biopsy-proven mature teratoma in anterior mediastinum (arrow) showing loss of fat planes and $>90^{\circ}$ angle of contact with superior vena cava (SVC) and ascending aorta. (B) Limited cine MRI axial images of 11-year-old boy with biopsy-proven mature teratoma in end-systolic (B) and end-diastolic (C) phases show the presence of chemical shift artifact and differential sliding motion between the mass and cardiovascular structures. Sliver of pericardial fat can be appreciated between SVC, ascending aorta and the mass in the end systolic image, that is, absent in end-diastolic image suggesting presence of sliding motion

areas of the body like delineating renal borders in abdominal imaging; identification of fat in lipomas, angiomyolipomas in the liver or kidneys and in identifying lipid-rich adrenal adenomas. In this study, we have used this artifact to identify the presence of fat plane between mediastinal masses and cardiovascular structures. However, radiologists should be aware that the chemical shift artifact can be minimized or accentuated by altering various imaging parameters like the field of view, frequency-encoding direction and the receiver frequency bandwidth. Choosing the frequency-encoding direction in the plane with the narrowest lipid-water interface, decreasing the field of view and increasing the frequency bandwidth can minimise the chemical shift artefact and vice versa. ${ }^{[1]}$ These things should be kept in mind while using chemical shift artifact as a sign, to avoid misinterpretation.

Our study shows the superiority of MR imaging over CT in identifying preserved fat planes between abutting structures using the presence of type 2 chemical shift artefact in cine and non-cine SSFP sequences. Out of 137 cardiovascular structures displaying loss of fat plane on CECT, 106 structures showed the presence of a chemical shift artifact suggesting the presence of fat-water interface and out of those 97 structures were not invaded intraoperatively.

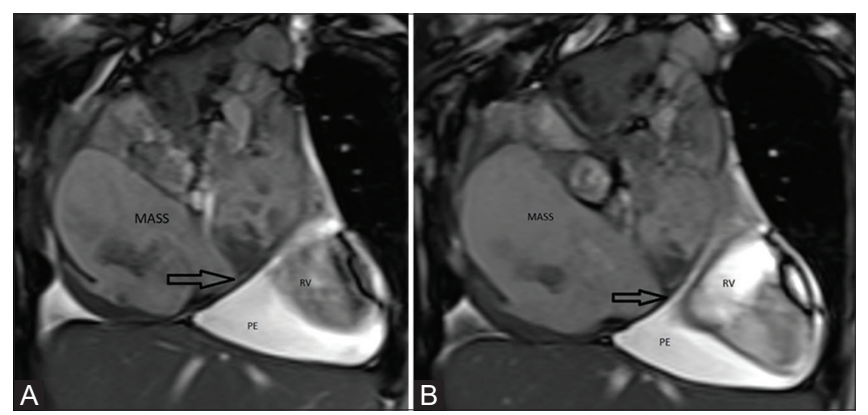

Figure 7 ( $A$ and $B$ ): (A) Limited Cine coronal images of a 32-year-old lady with biopsy-proven mature teratoma in end-systolic $(A)$ and end-diastolic phases (B) show a large mediastinal mass with a broad surface of contact with the pericardium and moderate pericardial effusion. There is a loss of sliding motion between the pericardium and the mediastinal mass. However, chemical shift artifact is maintained (arrow) suggesting no invasion on MRI. Intraoperatively, there was no invasion and loss of sliding motion was due to the associated pericardial effusion

We found that the presence of sliding motion can be used to rule out cardiovascular invasion with a high degree of confidence. This was particularly true while assessing the cardiac chambers, as the differential sliding motion is more pronounced in the normal cardiac chambers. ${ }^{[16]}$ Mediastinal vessels, especially those that are away from the heart like descending thoracic aorta, IVC, brachiocephalic vein and pulmonary veins, normally show minimum sliding motion, hence, leading to a false interpretation of sliding motion. Out of 12,9 (75\%) cases where MRI findings did not correlate with intraoperative findings were related to mediastinal vessels. Large intrathoracic masses can reduce the extent of differential motion between abutting structures due to the mass effect resulting in false negative cases. ${ }^{[17]}$ We found that pericardial effusion is another factor that can affect sliding motion, as seen in one of our cases where the differential motion between pericardium and mass was absent due to massive pericardial effusion rather than actual invasion. To summarize, we feel the following factors may lead to false interpretation of sliding motion on cine MRI; mediastinal vessels that are away from the heart, large and bulky mediastinal masses, gross pericardial effusion (limiting the motility of pericardium) and inflammatory adhesions between the mass and adjacent cardiovascular structures.

Some previous studies evaluated the effectiveness of cine MRI in assessing cardiovascular invasion by mediastinal masses. A study conducted by Seo et al. in 2005 evaluated the presence of sliding motion in 39 cardiovascular structures in 20 patients with accuracy of $94.4 \% .{ }^{[16]}$ Ong et al. in 2016 assessed sliding motion in 48 cardiovascular structures with accuracy, sensitivity and specificity of $88.5 \%, 70 \%$, and $97.6 \%$, respectively. ${ }^{[18]}$ Both studies also assessed the presence of intervening fat planes between mediastinal mass and cardiovascular structure using T1WI and T2WI. None of 
the above studies looked at the role of type 2 chemical shift artefact in excluding invasion of cardiovascular structures.

After a trial with few cases and realizing the potential benefits, the authors now routinely clinically use cine MRI as a problem-solving tool for any doubt of cardiovascular invasion by mediastinal masses on CT.

MRI-tagging sequences have been used for the diagnosis of constrictive pericarditis by looking at adherence and immobility of the myocardial-pericardial interface. ${ }^{[19,20]}$ These sequences use a grid-like pattern of saturated areas over an image slice and allow the study of the deformation of the grids with cardiac cycle. To our knowledge, these sequences have not been used in the context of the invasion of cardiovascular structures by mediastinal masses. We propose that these sequences can be used in future studies.

The authors are aware of some of the limitations of the study. Firstly, this was a retrospective study with its associated limitations. The accuracy of imaging findings involving 25 cardiovascular structures in 7 patients who were not operated owing to the advanced stage of disease or distant metastases, could not be assessed, hence, these structures had to be excluded from the study.

\section{Conclusion}

SSFP cine MRI can be used as an effective complementary imaging modality in the evaluation of cardiovascular invasion by mediastinal masses and help in planning the future course of management. The presence of type 2 chemical shift artifact and differential sliding motion between the mass and cardiovascular structure can effectively exclude invasion, thus facilitating surgery in patients with equivocal $\mathrm{CT}$ findings.

\section{Financial support and sponsorship}

Nil.

\section{Conflicts of interest}

There are no conflicts of interest.

\section{References}

1. Herman SJ, Winton TL, Weisbrod GL, Towers MJ, Mentzer SJ. Mediastinal invasion by bronchogenic carcinoma: CT signs. Radiology 1994;190:841-6.

2. Quint LE, Glazer GM, Orringer MB. Central lung masses: Prediction with CT of need for pneumonectomy versus lobectomy. Radiology 1987;165:735-8.

3. Glazer HS, Kaiser LR, Anderson DJ, Molina PL, Emami B, Roper CL, et al. Indeterminate mediastinal invasion in bronchogenic carcinoma: CT evaluation. Radiology 1989;173:37-42.
4. Martini N, Heelan R, Westcott J, Bains MS, McCormack P, Caravelli J, et al. Comparative merits of conventional, computed tomographic, and magnetic resonance imaging in assessing mediastinal involvement in surgically confirmed lung carcinoma. J Thorac Cardiovasc Surg 1985;90:639-48.

5. Rendina EA, Bognolo DA, Mineo TC, Gualdi GF, Caterino M, Di Biasi C, et al. Computed tomography for the evaluation of intrathoracic invasion by lung cancer. J Thorac Cardiovasc Surg 1987;94:57-63.

6. Bacha EA, Chapelier AR, Macchiarini P, Fadel E, Dartevelle PG. Surgery for invasive primary mediastinaltumors. Ann Thorac Surg 1998;66:234-9.

7. Puderbach M, Hintze C, Ley S, Eichinger M, Kauczor H-U, Biederer J. MR imaging of the chest: A practical approach at 1.5T. Eur J Radiol 2007;64:345-55.

8. Schvartzman PR, White RD. Imaging of cardiac and paracardiac masses. J Thorac Imaging 2000;15:265-73.

9. Batra P, Brown K, Collins JD, Holmes EC, Steckel RJ, Shapiro BJ. Mediastinal masses: Magnetic resonance imaging in comparison with computed tomography. J Natl Med Assoc 1991;83:969-74.

10. Takahashi K, Al-Janabi NJ. Computed tomography and magnetic resonance imaging of mediastinaltumors. J Magn Reson Imaging 2010;32:1325-39.

11. Steady-State MR Imaging Sequences: Physics, Classification, and Clinical Applications I RadioGraphics [Internet]. Available from: https://pubs.rsna.org/doi/full/10.1148/rg.284075031. [Last cited on 2020 Jun 08].

12. Gradient echo imaging - Mark1 - 2012 - Journal of Magnetic Resonance Imaging - 20 Wiley Online Library [Internet]. Available from: 21 https://onlinelibrary.wiley.com/doi/full/10.1002/ jmri.23638. [Last cited on 2020 Jun 08].

13. Hashemi RH, Bradley WG, Lisanti CJ, Ovid Technologies I. MRI: The Basics [Internet]. $2^{\text {nd }}$ ed. Philadelphia: Lippincott Williams \& Wilkins; 2004. Available from: https://trove.nla.gov.au/ version/46500853. [Last cited on 2020 May 22].

14. Hood MN, Ho VB, Smirniotopoulos JG, Szumowski J. Chemical shift: The artifact and clinical tool revisited. Radiogr Rev Publ Radiol Soc N Am Inc 1999;19:357-71.

15. Ma J. Dixon techniques for water and fat imaging. J Magn Reson Imaging 2008;28:543-58.

16. Seo JS, Kim YJ, Choi BW, Choe KO. Usefulness of magnetic resonance imaging for evaluation of cardiovascular invasion: Evaluation of sliding motion between thoracic mass and adjacent structures on cine MR images. J Magn Reson Imaging 2005;22:234-41.

17. Kajiwara N, Akata S, Uchida O, Usuda J, Ohira T, Kawate N, et al. Cine MRI enables better therapeutic planning than $\mathrm{CT}$ in cases of possible lung cancer chest wall invasion. Lung Cancer Amst Neth 2010;69:203-8.

18. The Diagnostic Utility of Cardiac-Gated Magnetic Resonance Imaging for Assessing Surgical Resectability of Mediastinal Tumors [Internet]. Available from: https://e-cvia.org/search. php? where $=$ aview\&id $=10.22468 /$ cvia $.2016 .00038 \&$ code $=9989$ CVI A\&vmode=PUBREADER. [Last cited on 2020 May 22].

19. Power JA, Thompson DV, Rayarao G, Doyle M, Biederman RWW. Cardiac magnetic resonance radiofrequency tissue tagging for diagnosis of constrictive pericarditis: A proof of concept study. J Thorac Cardiovasc Surg 2016;151:1348-55.

20. Kojima S, Yamada N, Goto Y. Diagnosis of constrictive pericarditis by tagged cine magnetic resonance imaging. $\mathrm{N}$ Engl J Med 1999;341:373-4. 